\title{
Seroprevalencia de leptospirosis y factores asociados en trabajadores del servicio de aseo urbano de la Municipalidad de Asunción, Paraguay
}

\author{
Francisco López, Margarita Samudio, Dalva María de Assis y Águeda Cabello
}

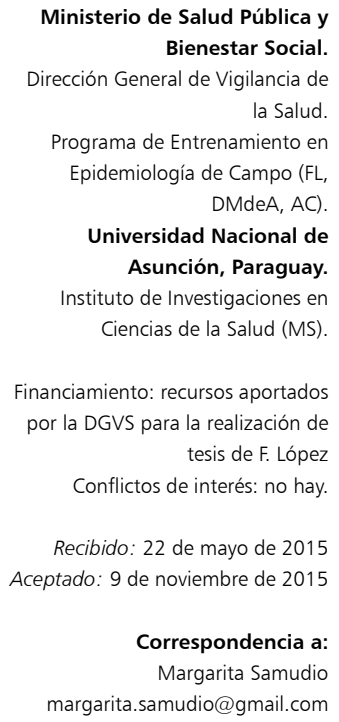

Financiamiento: recursos aportados por la DGVS para la realización de tesis de F. López

Conflictos de interés: no hay.

Recibido: 22 de mayo de 2015 Aceptado: 9 de noviembre de 2015

Correspondencia a: Margarita Samudio margarita.samudio@gmail.com

\section{Seroprevalence of leptospirosis and associated factors in workers of the urban cleaning service of the Municipality of Asuncion, Paraguay}

Introduction: Leptospirosis is a serious infectious disease that has been linked to occupational activities such as farmers, sewer workers, garbage collectors, butchers and veterinarians. It is acquired through contact with major reservoirs like rodents, pets, or the environment contaminated by their urine. In Paraguay, there are no published reports on the seroprevalence of leptospirosis related to work activity. Objective: To determine the seroprevalence and factors associated with transmission of leptospirosis in urban sanitation workers. Methodology: Descriptive transversal study. The study included workers from the Department of Urban Cleanliness of the City of Asuncion, who had completed at least one month on the job and who agreed to participate in the study. Sampling was stratified and proportional to the number of workers. After informed consent was obtained, participants were surveyed and a blood sample was taken for detection of Leptospira IgG antibodies by ELISA. Results: Leptospirosis seroprevalence was 8.6\% (29/339), all positive cases were male and prevalence was statistically higher in workers of the collection area than in those of other work areas. Conclusion: We found a lower than expected seroprevalence. However, conditions observed at work and at home are conducive to transmission of infection. We recommend the improvement of prevention strategies and health promotion in this population.

Key words: Seroprevalence, leptospirosis, sanitation, Paraguay.

Palabras clave: Seroprevalencia, leptospirosis, trabajadores, aseo urbano, Paraguay.

\section{Introducción}

L a leptospirosis es una enfermedad infecciosa grave que puede causar grandes epidemias, tiene una distribución mundial y un comportamiento endémico en muchos países ${ }^{1}$. En estas regiones, los cambios climáticos e inundaciones, además de los niveles sanitarios deficientes y abundante población de roedores, constituyen importantes determinantes de la infección ${ }^{2}$.

Existe un alto riesgo ocupacional de contraer la enfermedad $^{3}$, vinculada principalmente con actividades como la agricultura, trabajos en alcantarillas, recolección de basuras, carnicerías y manejo de animales ${ }^{4}$. Los trabajadores de los servicios de aseo urbano están constantemente expuestos a contraer enfermedades infecciosas, sobre todo si no utilizan equipos de protección personal para realizar sus actividades o por desconocimiento del modo de transmisión de enfermedades como la leptospirosis. En operarios de aseo en la región del Caribe colombiano se demostró una prevalencia de 13,6\% de infección ${ }^{5}$. También, existen varios estudios extranjeros que han reportado infecciones asociadas a actividades recreacionales o deportivas ${ }^{6-9}$.
Paraguay no cuenta con información suficiente sobre la situación de esta enfermedad y no dispone de estudios publicados sobre la seroprevalencia de leptospirosis relacionada a la actividad laboral. Por lo tanto, existe la necesidad de establecer la seroprevalencia y conocer los factores ocupacionales relacionados a la transmisión de la leptospirosis en los trabajadores recolectores de basuras, de manera de planificar y formular intervenciones para la vigilancia, prevención y control de la enfermedad. El objetivo del estudio fue determinar la seroprevalencia y los posibles factores asociados a la transmisión de Leptospira sp en los trabajadores de los servicios urbanos (áreas verdes, aseo urbano y recolección de residuos) de la Municipalidad de Asunción, Paraguay, en el año 2013.

\section{Material y Métodos}

\section{Tipo y período de estudio}

Estudio observacional descriptivo de corte transversal, con componente analítico, realizado entre los meses de marzo de 2013 y junio de 2014 en la ciudad de Asunción, Paraguay. 


\section{Población de estudio}

La población blanco de este estudio estuvo compuesta por los trabajadores pertenecientes a la Dirección de Servicios Urbanos de la Municipalidad de Asunción, acá definidos por trabajadores recolectores de basuras, registrados en la nómina de contratados y permanentes en el año de 2013, y que aceptaron participar del estudio.

Los trabajadores recolectores de basuras están especificados de acuerdo a las funciones y actividades que realizan en relación a la recolección de basura y se clasifican en tres dependencias de trabajo: Áreas verdes: encargados de la limpieza de los parques, plazas y podado de árboles; Aseo urbano: encargados de recolectar la basura de las calles de los diferentes contenedores o basureros municipales que se encuentran fuera del mercado y lugares comerciales y Recolección de residuos: encargados de recolectar las basuras de los domicilios.

\section{Muestra}

Para este estudio fue realizado un muestreo aleatorio, estratificado por dependencia y área de trabajo. Para el cálculo de tamaño de la muestra se utilizó el programa Epi-Info 3.5.3 (CDC, Atlanta), para un error máximo de $5 \%$ y una prevalencia esperada de la infección por Leptospira sp de $50 \%$ en la población de estudio y un nivel de confianza de $95 \%$.

De una población de 1.343 trabajadores, distribuidos en áreas verdes 150 (11\%), aseo urbano 886 (66\%) y recolección residuos 307 (23\%), por la técnica de muestreo propuesta, se planificó incluir a 299, sumado a $10 \%$ de posible rechazo para la colecta de muestra biológica, totalizando 328 trabajadores. Una vez obtenido el número de trabajadores a incluir en el estudio se estratificó proporcionalmente, de acuerdo al número de personas existentes a cada dependencia, y al final se hizo un sorteo con el listado nominal del personal a ser encuestado por área.

\section{Criterios de inclusión}

Como criterio de inclusión se consideró a todos los trabajadores con antigüedad mayor a un mes que estuviesen realizando sus funciones laborales en el mes que se realizó la encuesta y que aceptaron voluntariamente a participar del estudio.

\section{Recolección de datos}

Para la encuesta se utilizó un cuestionario semiestructurado en formato electrónico, en un software libre denominado Magpi ${ }^{\circledR}$, que es un generador de encuestas electrónicas, compatible con Android, iPhone OS y Symbian. Los datos fueron exportados directamente por internet o se puede realizar una exportación en la memoria de teléfonos celulares o tablets para luego ser recuperado en una planilla Excel ${ }^{\circledR}$. El cuestionario estuvo compuesto de variables socio-demográficos, características de la vivienda, riesgo ambiental de la vivienda, circunstancias relacionadas al trabajo, protección personal, antecedentes clínicos referentes a la enfermedad y resultado de laboratorio.

\section{Toma de muestra y análisis de laboratorio}

Se tomó $5 \mathrm{ml}$ de sangre de la vena cubital a cada encuestado, se extrajo el suero y posteriormente se almacenó a $-20^{\circ} \mathrm{C}$, hasta su análisis mediante el método de ELISA Leptospira IgG (EIA-4245®), DRG International Inc., U.S.A.) siguiendo las indicaciones del fabricante. La encuesta y la extracción de sangre se realizaron en los diferentes horarios de trabajo (mañana, tarde y noche).

\section{Análisis y gestión de datos}

Los resultados son presentados en forma de frecuencias absolutas y relativas. En el análisis bivariado se utilizó la prueba estadística $\chi^{2}$ para la evaluación de la asociación de las variables cualitativas con la presencia de anticuerpos anti-leptospira a un nivel de significación estadística de 0,05 . Además se calculó el odds ratio (OR) con su intervalo de confianza de $95 \%$.

\section{Asuntos éticos}

El protocolo de investigación fue aprobado por el Comité de Ética del Hospital de Clínicas de la Universidad Nacional de Asunción. Se obtuvo el consentimiento informado de las personas que resultaron seleccionadas, una vez que se les explicó la naturaleza del estudio. Para asegurar la confidencialidad de la información de las personas, los datos colectados en este estudio fueron manejados en forma de códigos y almacenados en una computadora a la que, sólo tenían acceso los investigadores. Los riesgos del estudio fueron reducidos al mínimo, la toma de la muestra fue realizada por profesionales de salud capacitados, que informaban a los participantes los posibles riesgos. A los casos positivos se les explicó sobre el tratamiento en esta fase de la enfermedad con una entrevista individual en el dispensario médico perteneciente al MSP y BS que se encuentra en las dependencias de su lugar de trabajo.

\section{Resultados}

En el presente estudio fueron incluidos 339 trabajadores del área urbana de la Municipalidad de Asunción, entre 21 a 70 años de edad (edad promedio: 43,9 años; DS 9,4 años). La distribución de los encuestados por grupo etario y antigüedad se presenta en la Tabla 1.

Se observó predominancia del sexo masculino (n: 317 ; 93,5\%). Escolaridad: 93/339 (27,4\%) tenía el nivel primario completo y $85(25,1 \%)$ nivel secundario incompleto. Actividad laboral: $106(31,3 \%)$ eran choferes, $96(28,3 \%)$ 


\begin{tabular}{|c|c|c|}
\hline \multicolumn{3}{|c|}{$\begin{array}{l}\text { Tabla 1. Características socio-demográficas de los } \\
\text { trabajadores de la Dirección de Aseo Urbano de la } \\
\text { Municipalidad de Asunción, Paraguay (n: 339) }\end{array}$} \\
\hline Características socio-demográficas & $\mathbf{n}$ & $\%$ \\
\hline Edad en años (promedio $\pm \mathrm{DE}$ ) & $43,9 \pm 9$ & \\
\hline \multicolumn{3}{|l|}{ Grupo etario (años) } \\
\hline 20 a 40 & 123 & 36,3 \\
\hline 41 a 60 & 204 & 60,2 \\
\hline$>60$ & 12 & 3,5 \\
\hline \multicolumn{3}{|l|}{ Antigüedad en el trabajo (años) } \\
\hline$<1$ & 6 & 1,8 \\
\hline 1 a 5 & 40 & 11,8 \\
\hline 6 a 10 & 34 & 10 \\
\hline$>10$ & 259 & 76,4 \\
\hline
\end{tabular}

Tabla 2. Seropositividad según presencia de factor de riesgo domiciliario de los trabajadores de la Dirección de Aseo Urbano Asunción, Paraguay 2013

\begin{tabular}{|lrlrlll}
\hline Factor de riesgo (FR) & $\begin{array}{c}\text { Seropositivo/ } \\
\text { presencia de FR }\end{array}$ & $\begin{array}{c}\text { Seropositivo/ } \\
\text { ausencia de FR }\end{array}$ & IC $_{95 \%}$ OR & Valor $\mathbf{p}$ \\
Sexo masculino & $29 / 317$ & $(9,1 \%)$ & $0 / 22$ & $(0 \%)$ & $1,06-1,13$ & 0,13 \\
Edad 20 a 40 años & $10 / 123$ & $(8,1 \%)$ & $19 / 216$ & $(8,8 \%)$ & $0,44-1,92$ & 0,83 \\
\hline Distancia casa-río 100-300 m & $7 / 68(10,3 \%)$ & $22 / 271$ & $(8,1 \%)$ & $0,93-1,11$ & 0,55 \\
Distancia casa-vertedero 100-300 m & $4 / 29(13,8 \%)$ & $25 / 310$ & $(8,1 \%)$ & $0,91-1,23$ & 0,29 \\
Casa sufre inundaciones & $3 / 47$ & $(6,4)$ & $26 / 292$ & $(8,9 \%)$ & $0,89-1,05$ & 0,56 \\
Tiene perros & $18 / 256$ & $(7,0 \%)$ & $11 / 83$ & $(13,3 \%)$ & $0,85-1,02$ & 0,07 \\
Tiene gatos & $8 / 110$ & $(7,3)$ & $21 / 229$ & $(9,2 \%)$ & $0,91-1,04$ & 0,34 \\
Tiene cerdos & $1 / 15$ & $(6,7 \%)$ & $28 / 324$ & $(8,6)$ & $0,85-1,12$ & 0,78 \\
Vio roedores & $19 / 229$ & $(8,3)$ & $10 / 110$ & $(9,1 \%)$ & $0,92-1,06$ & 0,80 \\
Mató o estuvo en contacto con roedores & $14 / 172$ & $(8,1)$ & $15 / 167$ & $(19,0 \%)$ & $0,92-1,05$ & 0,78 \\
\hline
\end{tabular}

Tabla 3. Seropositividad según presencia de factor de riesgo ocupacional en trabajadores de la Dirección de Aseo Urbano Asunción, Paraguay 2013

\begin{tabular}{|c|c|c|c|c|}
\hline Factor de riesgo (FR) & $\begin{array}{l}\text { Seropositivo/ } \\
\text { presencia de FR }\end{array}$ & $\begin{array}{l}\text { Seropositivo/ } \\
\text { ausencia de FR }\end{array}$ & $\mathrm{IC}_{95 \%} \mathrm{OR}$ & Valor $p$ \\
\hline Trabaja en el área de recolección & $14 / 86(16,3 \%)$ & $15 / 253 \quad(5,9 \%)$ & $1,02-1,24$ & 0,002 \\
\hline $\begin{array}{l}\text { Recibió capacitación sobre uso de } \\
\text { equipo de protección personal }\end{array}$ & $10 / 81(12,3 \%)$ & $19 / 258 \quad(7,4 \%)$ & $0,96-1,15$ & 0,16 \\
\hline Usa mascarilla & $0 / 6 \quad(0)$ & $29 / 304 \quad(8,7 \%)$ & $0,88-0,94$ & 0,44 \\
\hline Usa guantes & $14 / 141 \quad(9,9 \%)$ & $15 / 198 \quad(7,6 \%)$ & $0,95-1,09$ & 0,44 \\
\hline Alguna vez trabajó con pies descalzos & $12 / 98(11,5 \%)$ & $17 / 241 \quad(7,1 \%)$ & $0,96-1,13$ & 0,22 \\
\hline Suele ver roedores en su trabajo & $21 / 263 \quad(8,0 \%)$ & $8 / 76(10,5 \%)$ & $0,89-1,05$ & 0,48 \\
\hline Mató en su trabajo algún roedor & $7 / 105 \quad(6,7 \%)$ & $22 / 234 \quad(9,4 \%)$ & $0,90-1,03$ & 0,4 \\
\hline $\begin{array}{l}\text { Tuvo alguna lesión en las manos o } \\
\text { pies durante su trabajo }\end{array}$ & $12 / 136 \quad(8,8 \%)$ & $17 / 203 \quad(8,3 \%)$ & $0,94-1,07$ & 0,88 \\
\hline
\end{tabular}

eran barrenderos, $92(27,1 \%)$ recolectores y $45(13,3 \%)$ supervisores. Área de trabajo: $210(61,9 \%)$ eran de aseo urbano, $86(25,4 \%)$ de recolección y $43(12,7 \%)$ de áreas verdes. La mayoría, 259 (76,4\%), tenía una antigüedad en el trabajo de $>10$ años.

En relación a los factores de riesgo relacionados al domicilio del trabajador, 256 individuos $(75,5 \%)$ refirieron tener perros y $110(32,4 \%)$ gatos, otros animales como vacas y cerdos también fueron reportados. Con respecto a los roedores, 29 personas $(66,7 \%)$ afirmaron haberlos visto alguna vez alrededor de su casa, $172(50,7 \%)$ haberlos matado o haber estado en contacto con un roedor. Sesenta y ocho $(20,1 \%)$ indicaron que su casa se encontraba ubicada entre 100 y $300 \mathrm{~m}$ del río y $29(8,6 \%)$ su casa estaba entre 100 y $300 \mathrm{~m}$ del vertedero. Cuarenta y siete $(13,9 \%)$ refirieron que su casa sufría inundaciones por lluvia o desborde del río.

Con respecto a las circunstancias ligadas al trabajo, 258 trabajadores $(76,1 \%)$ refirieron no haber recibido capacitación sobre uso de equipo de protección personal, $104(30,7 \%)$ afirmaron no usar equipo de protección personal, $136(40,1 \%)$ presentaron alguna lesión en sus manos o pies durante su tiempo de trabajo, 98 (28,3\%) estuvieron en contacto con el agua o barro con los pies descalzos. Refirieron la presencia de roedores en el trabajo $263(77,6 \%)$ encuestados y $105(31 \%)$ lo mataron o estuvieron en contacto con ellos.

De los 339 trabajadores, 29 presentaron serología positiva para lestospirosis, siendo por lo tanto, la seroprevalencia global de 8,6\% (IC 95\%: 5,9\%-12,2\%). De los 29 casos positivos, $6,9 \%$ (n: 2) pertenecía a áreas verdes, $44,9 \%$ (n: 13 ) a aseo urbano y $48,2 \%$ (n: 14 ) a recolección de residuos domiciliarios. Todos los casos positivos eran del sexo masculino.

No se identificó factor de riesgo alguno, relacionado al domicilio del trabajador, con su estado de seropositividad (Tabla 2).

En relación a los factores de riesgo ocupacionales, la única circunstancia que se relacionó significativamente (valor p: 0,002 ) con la seropositividad de los trabajadores fue el de trabajar en el área de recolección de residuos domiciliarios, siendo la seroprevalencia en este grupo 16,3\% (IC 95\%: 9,2\%-25,8\%) en comparación con 5,9\% de los que trabajaban en otras áreas (Tabla 3).

\section{Discusión}

La seroprevalencia de leptospirosis en los trabajadores de servicios de aseo urbano de la Municipalidad de Asunción fue de 8,6\% (IC 95\%: 5,9\%-12,2\%). El valor encontrado en este estudio es mucho menor al reportado por Cabello y cols., en un estudio de seroprevalencia de dengue y leptospirosis realizado en una localidad ribereña al sur de Paraguay, en el cual se encontró una prevalencia 
de leptospirosis de $24,1 \%{ }^{10}$. La diferencia podría explicarse por el hecho de que en esta zona, la principal ocupación de los pobladores es el cultivo de arroz, en concordancia con lo reportado en Perú donde se habían relacionado algunas localidades arroceras con hiperendemia de leptospirosis y factores de riesgo asociados, además de las condiciones sanitarias precarias ${ }^{11}$. Es bien conocida que la infección está vinculada principalmente con actividades ocupacionales en los agricultores, los trabajos en alcantarillas y manejo de animales como veterinarios que son susceptibles de adquirir la infección debido a su alto riesgo de exposición ${ }^{12}$.

Existen varios estudios de seroprevalencia de leptospirosis que arrojan resultados con grandes variaciones debido a factores relacionados a la población estudiada, los métodos serológicos utilizados o la fase de la enfermedad investigada (IgG o IgM), entre otros. Los estudios de seroprevalencia realizados en los últimos 20 años encontraron resultados dispares, dependiendo de la zona y la ocupación; si el estudio fue hecho en la población general $^{13-16}$, en grupos de alto riesgo ${ }^{17-24} \mathrm{o}$ en contactos de enfermos $^{25}$. En general, la prevalencia de leptospirosis en países latinoamericanos está en alrededor de 1,2 a $6 \%{ }^{26,27}$. En un estudio realizado en Chile como país, se reportó una prevalencia muy baja $(0,4 \%)^{28}$. En oposición, en operarios de aseo en la región del Caribe colombiano la prevalencia fue mucho mayor $(13,6 \%)^{5}$.

La leptospirosis es una zoonosis raramente diagnosticada en Paraguay, probablemente por falta de conocimiento de la enfermedad por el personal médico, baja especificidad de los síntomas, que se confunden con patologías con sintomatologías similares y ausencia de métodos diagnósticos de la infección en los laboratorios públicos y privados. La vigilancia de laboratorio de leptospirosis entre los años 1999 y 2009 en el país, pudo identificar 55 muestras positivas de las 845 muestras analizadas.

Estudios realizados en Brasil en pacientes con dengue y hepatitis viral han demostrado que entre 9 y $15 \%$ de estos pacientes presentan además positividad para anticuerpos recientes anti leptospira ${ }^{29}$, sugiriendo que la sub-notificación puede ser aún más alta cuando en los casos existen dos patologías similares concomitantes como ocurre en el Paraguay.

A pesar de la seropositividad relativamente baja en esta población, existen muchas condiciones propicias para la transmisión de la infección, tanto en el domicilio como en el medio laboral. Entre las causas domiciliarias se puede mencionar el alto porcentaje de individuos que refieren tener animales en sus casas, como perros, gatos, además de vacas y cerdos. Con respecto a los roedores, es relativamente común que haya contactos con ellos, tanto alrededor de sus casas, como en el lugar de trabajo. Es importante dar a conocer a estos trabajadores la capacidad de la bacteria de penetrar la piel intacta ${ }^{2}$, considerando que muchos de los trabajadores refirieron haber trabajado con alguna lesión en sus manos o pies.

En relación a los riesgos ambientales, una proporción importante de los encuestados reportó condiciones favorables para adquirir la infección, como residir cerca del rio o del vertedero, y sufrir inundaciones por lluvia o desborde del río. Los factores de riesgo que se repiten en los diferentes estudios son: exposición a aguas contaminadas, manipulación de animales, contacto con animales, vivir en regiones con inundaciones frecuentes y áreas pobres y rurales ${ }^{30,31}$.

Llama la atención el alto porcentaje de personas que reportaron no haber recibido capacitación sobre uso de equipo de protección personal, sólo seis individuos refirieron usar mascarilla durante su trabajo, una importante proporción no utiliza equipo de protección personal y muchos reportaron haber trabajado con los pies descalzos. De ahí la importancia de implementar programas de educación continua en bioseguridad, no sólo para los trabajadores de mayor riesgo sino además, para el público en general en relación a los riesgos ocupacionales. Además de reducir los ambientes favorables para roedores responsables de mantener la transmisión.

La gran limitación del estudio fue la falta de identificación de los serovares circulantes. El método del estudio utiliza antígenos de la especie Leptospira biflexa (serovar Patoc 1) que ha demostrado tener la capacidad de detectar otros serovares. El único estudio que identificó los serovares circulantes en Paraguay fue producto de la vigilancia de laboratorio de leptospirosis entre los años 1999 y 2009 mostrando los siguientes serovares: tarassovi $(30 \%)$, autummalis (26\%), andamana (24\%), javanica y shermani $(17 \%)$, entre los más frecuentes.

Otra limitación del estudio es que sólo se midió la presencia de anticuerpos, los que pueden permanecer por años luego de la infección, y no confirmar el diagnóstico de la enfermedad. Se ha demostrado que los humanos pueden ser portadores de los anticuerpos por varios años luego de haber padecido la enfermedad. Esto hace difícil predecir factores de riesgo en esta muestra, ya que no se cuenta con datos primarios recolectados para este fin. Sólo permite comparar los datos descritos en esta serie de casos con los factores de riesgo reportados en la literatura científica.

En este estudio se utilizó la detección de anticuerpos a través de la técnica ELISA, con una sensibilidad de 86,5\% y una especificidad de $97 \%{ }^{31}$. La prueba de referencia es la prueba de aglutinación microscópica (microaglutinación en tubo-MAT) por su alta sensibilidad y especificidad, la que detecta anticuerpos contra serovares específicos; no obstante, presenta reacción cruzada con otros serovares y requiere el uso de sueros pareados que confirmen el aumento en los títulos, además del mantenimiento de cepas 
vivas susceptibles de contaminación, siendo necesaria su realización por laboratorios especializados ${ }^{32,33}$.

A pesar de que se observaron factores ambientales relacionados a la infección, no se identificó algún factor de riesgo relacionado al domicilio del trabajador y su estado de seropositividad. Esto probablemente se deba a que estos trabajadores comparten además de las condiciones físicas, ambientales y geográficas, los factores predisponentes para adquirir la infección. En relación a los factores de riesgo ocupacionales, la única circunstancia que se relacionó significativamente con la seropositividad de los trabajadores fue el de trabajar en el área de recolección de desechos domiciliarios. Esto podría deberse a una combinación de varios factores que no pudieron identificarse en forma individual ${ }^{30,31}$.

En conclusión, el estudio permitió establecer la seroprevalencia de anticuerpos anti-leptospira para esta población, categorizándola como de relativa baja prevalencia. A pesar de ello, las condiciones observadas, tanto en el lugar de trabajo como en el domicilio, son propicias para la transmisión de la infección por lo que se recomienda mejorar las medidas preventivas y de promoción de la salud en esta población.

Agradecimientos. A Marco Benítez por su colaboración en los trabajos de laboratorio.

\section{Resumen}

Introducción: La leptospirosis es una enfermedad infecciosa grave que ha sido vinculada con actividades ocupacionales, en los agricultores, trabajadores de alcantarillas, recolectores de basura, carniceros y veterinarios; se adquiere a través del contacto con sus principales reservorios como roedores, animales domésticos o ambiente contaminado por su orina. En Paraguay no se tiene reportes publicados sobre la seroprevalencia de leptospirosis relacionados a la actividad laboral. Objetivo: Determinar la seroprevalencia y factores laborales asociados a la transmisión de la leptospirosis en trabajadores de aseo urbano. Metodología: Estudio descriptivo de corte transversal, en el que fueron incluidos trabajadores del Departamento de Aseo Urbano de la Municipalidad de Asunción, con una antigüedad mínima de un mes y que aceptaron participar del estudio. El muestreo fue probabilístico estratificado, proporcional al número de trabajadores. Previo consentimiento informado, los participantes fueron encuestados, y se extrajo una muestra de sangre para la detección de anticuerpos del tipo IgG anti Leptospira por el método de ELISA. Resultados: La seroprevalencia de leptospirosis fue de $8,6 \%$ (29/339), todos los casos positivos fueron del sexo masculino, los trabajadores del área de recolección tuvieron una prevalencia estadísticamente mayor que aquellos de otras áreas de trabajo. Conclusión: Se encontró una seroprevalencia menor a la esperada. Sin embargo, a pesar de la prevalencia relativamente baja, las condiciones observadas, tanto en el trabajo como en el hogar, son propicias para la transmisión de la infección por lo que se recomienda mejorar las medidas preventivas y de promoción de la salud en esta población.

\section{Referencias bibliográficas}

1.- Pappas G, Papadimitriou P, Siozopoulou V, Christou L, Akritidis N. The globalization of leptospirosis: worldwide incidence trends. Int J Infect Dis 2008; 12 (4): 351-7. [PubMed: 18055245].

2.- Evangelista K V, Coburn J. Leptospira as an emerging pathogen: a review of its biology, pathogenesis and host immune responses. Future Microbiol 2010; 5 (9): 1413-25. doi: 10.2217/fmb.10.102.

3.- Bharti A R, Nally J E, Ricaldi J N, Matthias M A, Díaz M M, Lovett M A, et al. Leptospirosis: a zoonotic disease of global importance. Lancet Infect Dis 2003; 3 (12): 757-71.

4.- World Health Organization and International Leptospirosis Society. Human leptospirosis: guidance for diagnosis, surveillance and control 2003; http://whqlibdoc.who.int/hq/2003/WHO_ CDS CSR EPH 2002.23.pdf.

5.- Nájera S, Alvis N, Babilonia D, Álvarez L, Máttar S. Leptospirosis ocupacional en una región del Caribe colombiano. Salud Pública
Méx 2005 junio [citado el 20 de septiembre de 2015]; 47 (3): 240-4.

6.- Morgan J, Bornstein S L, Karpati A M, Bruce M L, Bolin C A, Austin C C, et al. Outbreak of leptospirosis among triathlon participants and community residents in Springfield, Illinois, 1998. Clin Infect Dis 2002; 34: 1593-9.

7.- Victoriano A F, Smythe L D, Gloriani-Barzaga N, Cavinta L L, Kasai T, Limpakarnjanarat K, et al. Leptospirosis in the Asia Pacific region. BMC Infect Dis 2009; 9: 147.

8.- Sejvar J, Bancroft E, Winthrop K, Bettinger J, Bajani M, Bragg S, et al. Leptospirosis in "Eco-challenge" Athletes, Malaysian Borneo, 2000. Emerg Infect Dis 2003; 9 (6): 702-7.

9.- Narita M, Fujitani S, Haake D A, Patterson D L. Leptospirosis after recreational exposure to water in the yaeyama islands, Japan. Am J Trop Med Hyg 2005; 73 (4): 652-6.

10.- Cabello A, Cabral M, Samudio M, Páez M, Jiménez R, Arce M, et al. Dengue y leptospirosis compartiendo el mismo nicho ecológico en la localidad ribereña de Carmen del Paraná (Itapúa). Mem Inst Investig Cienc Salud 2010 junio 2010, [citado el 19 de agosto de 2013]; 8 (1): 35-40.

11.- Cruz M R, Fernández V F, Arévalo R H. Hiperendemicidad de leptospirosis y factores de riesgo asociados en localidades arroceras del departamento de San Martín-Perú. Rev Perú Med Exp Salud Pública 2002; 19 (1): 10-6.

12.- Angnani R, Pathak A, Mishra M. Prevalence of leptospirosis in various risk groups. Indian J Med Microb 2003; 21: 1-5.

13.- Morshed M G, Konishi H, Terada Y, Arimitsu Y, Nakazawa T. Seroprevalence of leptospirosis in a rural flood prone district of Bangladesh. Epidemiol Infect 1994; 112 (3): 527-31.

14.- Navarrete-Espinosa J, Acevedo-Vales J A, Huerta-Hernández E, Torres-Barranca J, Gavaldón-Rosas D G. Prevalence of dengue and leptospira antibodies in the state of Veracruz, México. Salud Pública Mex 2006; 48 (3): 220-8.

15.- Cacciapuoti B, Ciceroni L, Pinto A, Apollini M, Rondinella V, Bonomi U, et al. Survey on the 
prevalence of leptospira infections in the Italian population. Eur J Epidemiol 1994; 10 (2): 17380.

16.- Kawaguchi L, Sengkeopraseuth B, Tsuyuoka R, Koizumi N, Akashi H, Vongphrachanh P, et al. Seroprevalence of leptospirosis and risk factor analysis in flood-prone rural areas in Lao PDR. Am J Trop Med Hyg 2008; 78 (6): 957-61.

17.- Perret C, Abarca K, Dabanch J, Solari V, García $\mathrm{P}$, Carrasco S, et al. Prevalencia y presencia de factores de riesgo de leptospirosis en una población de riesgo de la Región Metropolitana. Rev Med Chile 2005; 133: 426-31.

18.- Thai K T, Binh T Q, Giao P T, Phuong H L, Hung le Q, Van Nam N, et al. Seroepidemiology of leptospirosis in southern Vietnamese children. Trop Med Int Health 2006; 11 (5): 738-45.

19.- Swapna R N, Tuteja U, Nair L, Sudarsana J. Seroprevalence of leptospirosis in high risk groups in Calicut, North Kerala, India. Indian J Med Microbiol 2006; 24: 349-52.

20.- Lévesque B, Messier V, Bonnier-Viger Y, Couillard M, Côté S, Ward B J, et al. Seroprevalence of zoonoses in a Cree community (Canada). Diagn Microbiol Infect Dis 2007; 59 (3): 283-6.

21.- Gonçalves D D, Teles P S, dos Reis C R, Lopes F M, Freire R L, Navarro I T, et al. Seroepidemiology and occupational and environmental variables for leptospirosis, brucellosis and toxoplasmosis in slaughterhouse workers in the Paraná State, Brazil. Rev Inst Med Trop Sao Paulo 2006; 48 (3): 135-40.

22.- Riedemann S, Zamora J. Aspectos epidemiológicos de leptospirosis humana en el medio rural. Zbl Vet Med B 1982; 29: 702-7.

23.- Zamora J, Riedemann S, Montecinos M I, Cabezas X. Encuesta serológica de leptospirosis humana en ocupaciones de alto riesgo en Chile. Rev Med Chile 1990; 118: 247-52.

24.- Zamora J, Riedemann S, Leal H, Jackson C. Evidencia serológica de leptospirosis en labradores de arrozales. Rev Med Chile 1988; 116: 723-8.

25.- Silva H R, Tavares-Neto J, Bina J C, Meyer $R$. Leptospiral infection and subclinical presentation among children in Salvador, Bahia. Rev Soc Bras Med Trop 2003; 36 (2): 227-33.

26.- Platts-Mills J A, LaRochelle P, Campos K, Vinetz J M, Gotuzzo E, Ricaldi J N. Seroprevalence of leptospirosis in Puente Piedra, Lima, in 2006. Rev Perú Med Exp Salud Pública 2011; 28 (2): 273-6.

27.- Romero M H, Sánchez J A, Hayek L C. The prevalence of antibodies against Leptospira in urban human and canine populations from the Tolima Department. Rev Salud Pública (Bogotá) 2010; 12 (2): 268-75.
28.- Terrazas S, Olea A, Riedemann S, Torres M. Prevalencia de leptospirosis en adultos Chile, 2003. Rev Chilena Infectol 2012; 29 (6): 641-7.

29.- de Souza A I, Nogueira J M, Pereira M M. AntiLeptospira antibodies in patients in the State of Mato Grosso do Sul with clinical suspicion of dengue or viral hepatitis. Rev Soc Bras Med Trop 2007; 40 (4): 431-5.

30.- Carneiro M, Giacomini M L, Costa J M. Leptospirosis asociada a la exposición ocupacional: Estudio clínico y epidemiológico. Rev Chilena Infectol 2004; 21 (4): 339-44.

31.- Quiñonez M B, León M E, Fariña N, Falcón $M$. Vigilancia de la leptospirosis humana en el Laboratorio Central de Salud Pública en un período de 10 años (1999 a 2009). Rev. Par. Epidemiol. 2011; 2 (1): 41-8. Disponible en: http://www.vigisalud.gov.py/images/ documentos/revista/vol2\%20nro $\% 20$ nov2011\%20RPE/AO.\%20\%20QUINONEZ. pdf

32.- Bajan M D, Ashford D A, Bragg S L, Woods C W, Aye T, Spiegel R A, et al Evaluation of four commercially available rapid serologic tests for diagnosis of leptospirosis. J Med Microbiol 2003; 41 (2): 803-9.

33.- Levett P N. Leptospirosis. Clin Microbiol Rev 2001; 14: 296-326. 